\title{
Acute bilateral mass-occupying lesions in non-penetrating traumatic brain injury: a retrospective study
}

Yu Hu${ }^{1 \dagger}$, Hong Sun ${ }^{1+}$, Yanqing Yuan², Qiang Li', Siqing Huang ${ }^{1}$, Shu Jiang ${ }^{1}$, Kaili Liu ${ }^{3}$ and Chaohua Yang ${ }^{1 *}$

\begin{abstract}
Background: Traumatic acute bilateral mass-occupying lesions (TABML) is a common entity in head injury, with high morbidity and mortality. Our aim in this study was to evaluate the benefits of different treatment options and the outcome predictors in patients with TABML.

Methods: From October 2010 to November 2012, a consecutive cohort of patients aged 16-70 years with TABML were retrospectively analyzed based on the clinical and radiological characteristics. Patients with TABML were included if admitted within $24 \mathrm{~h}$ after injury and were excluded if they presented with infratentorial lesions, unilateral lesions within the first $24 \mathrm{~h}$ after injury, or penetrating head injury. According to their treatment option, patients were divided into three groups: a conservative treatment group, a unilateral surgery group, and a bilateral surgery group. Outcomes were assessed using the Glasgow Outcome Scale (GOS). Binary logistic regression analysis was applied to determine the outcome predictors.
\end{abstract}

Results: Forty-seven patients (58.8\%) had severe injuries (Glasgow Coma Scale score (GCS), 3-8) upon admission, and the overall mortality was $31.3 \%$ at 6 months post-injury. The mortality was $55.6 \%$ in patients who underwent conservative treatment $(\mathrm{N}=18), 17.9 \%$ in unilateral surgery patients $(\mathrm{N}=39)$, and $34.8 \%$ in the bilateral surgery group ( $N=23$ ). In the surgical group, the mortality was $53.3 \%$ (8 of 15) in those with a GCS of 3-5, which decreased steeply to $14.9 \%$ (7 of 47) of those with GCS $\geq 6$. On logistic regression analysis, the absence of pupillary reactivity, disappearances of basal cisterns and conservative treatment were related to higher mortality. A lower initial GCS score was associated with an unfavorable outcome. Midline shift tended to be associated with mortality and an unfavorable outcome, although statistical analysis did not show a significant difference.

Conclusions: TABML is suggestive of severe brain injury. As conservative treatment is always associated with a poorer outcome, surgery is advocated, especially in patients with a GCS score of $\geq 6$. Whereas the prognostic value of midline shift might be limited because of the counter-mass effect in TABML, the GCS score, the pupillary reactivity, and particularly, the compression of basal cisterns should be emphasized.

Keywords: Bilateral, Head injury, Lesions, Surgery, Traumatic brain injury

\footnotetext{
* Correspondence: yangchaohua999@126.com

${ }^{\dagger}$ Equal contributors

'Department of Neurosurgery, West China Hospital, Sichuan University, No

37, Guo Xue Xiang, Chengdu, Sichuan Province, China

Full list of author information is available at the end of the article
} 


\section{Background}

Traumatic brain injury (TBI) is a heterogeneous entity that encompasses several anatomical patterns, including epidural hematoma (EDH), subdural hematoma (SDH), hemorrhagic contusion ( $\mathrm{HC})$, diffuse axonal injury, subarachnoid hemorrhage, intraventricular hemorrhage, and diffuse brain swelling on the macroscopic level [1-4]. $\mathrm{EDH}, \mathrm{SDH}$, and $\mathrm{HC}$ are common types of acute mass-occupying lesions, which can be observed in various combinations (e.g., SDH with $\mathrm{HC}$ ), particularly in patients with severe head injury [2,5-7]. These massoccupying lesions may also present unilaterally or bilaterally on the computed tomography (CT) scans [8-10].

Traumatic acute bilateral mass-occupying lesions (TABML) is a common entity after contre-coup injury, with an estimated mortality ranging from $20 \%$ to 79\%; however, the optimal treatment options and the prognostic indicators for TABML are largely unknown [4,5,8-12]. Whether all patients with TABML will benefit from surgical intervention and, if surgery is performed, whether surgical intervention should be based on predominantly mass-occupying lesions (unilateral) or bilateral lesions [5,7-10,13], remain under discussion. Early prediction of outcomes is not only of great importance in clinical decision-making, but also is useful for stratification of patients for clinical trials and comparison of outcomes of different series [3,14]. The Glasgow coma scale (GCS) score on admission is a powerful independent predictor in TBI, a fact demonstrated in patients with TABML in previous studies [5,12]. The midline shift (MLS), another important predictor in TBI, is usually of less significance in bilateral than in unilateral abnormalities because of the counter-mass effect $[3-5,7,12,15]$. Whether the MLS and other predictors, such as the compression of basal cisterns, correlated with outcomes in TABML has not been extensively studied $[3,5]$.

In the study reported herein, our aim was to determine the benefits of different treatment options and the outcome predictors in patients with TABML.

\section{Methods}

\section{Patients}

Following approval by the medical ethics committee of the West China Hospital of Sichuan University (Chengdu, China), a consecutive cohort of patients aged 16-70 years with bilateral mass-occupying lesions after TBI was retrospectively enrolled and observed from October 2010 to November 2012. Patients were included if admitted within $24 \mathrm{~h}$ after injury. Exclusion criteria were defined as infratentorial lesions or only unilateral lesions within first $24 \mathrm{~h}$ after injury that subsequently developed into bilateral lesions [8]. Patients with penetrating head injury or any extracranial injuries that could affect outcomes were also excluded. Patients with limitations to surgical intervention, such as coagulopathy and Do Not Resuscitate (DNR) orders, were also excluded. The demographics, mechanism of injury, pupillary response, admission GCS score, CT characteristics, neurosurgical therapies, and Glasgow Outcome Scale (GOS) score at 6 months after injury were recorded.

\section{Computed tomography}

After initial resuscitation, a CT scan of the head was performed in all patients. MLS (in millimeters) was defined as displacement of the septum pellucidum. Traumatic mass-occupying lesions were noted and their volumes (in milliliters) estimated using the ellipsoid method described previously [3]. The largest lesion responsible for the primary symptoms was regarded as the predominant lesion; accordingly, patients were categorized as $\mathrm{EDH}$ with or without $\mathrm{HC}, \mathrm{SDH}$ with or without $\mathrm{HC}$, and pure $\mathrm{HC}$ as previously described [7], with minor modifications. We also scored the following variables on the initial head CT as described elsewhere: the status of basal cisterns (open, partially, and completely obliterated), traumatic subarachnoid hemorrhage (no blood, visible blood) and CT-visible deep lesions (no lesions, visible lesions) [15].

\section{Treatment}

Patients were treated according to guidelines for the management of severe head injury [16] and were divided into three groups based on their treatment option: conservative treatment group, unilateral surgery group, and bilateral surgery group. An intracranial pressure (ICP) probe was implanted in the lateral ventricle or intraparenchymally in 42 cases. Patients who had only ICP monitoring were included in the conservative group. Surgical indications were as follows: (1) signs of brain herniation; (2) clinical deterioration of consciousness and CT-confirmed hemorrhagic progression; (3) obvious mass-occupying effect and the compression of basal cistern; and (4) ICP $\geq 25 \mathrm{mmHg}$ after conservative treatment. The surgical procedures, unilateral or bilateral, were decided on by neurosurgeons according to clinical, radiological, and ICP findings. Operations were performed simultaneously if both lesions needed surgery, or sequentially (operations at separate times) if a significant contralateral lesion or uncontrolled intracranial hypertension (ICP $\geq 25 \mathrm{mmHg}$ ) developed after the primary operation developed.

\section{Outcome}

The GOS was assessed at 6 months after injury $[1,3]$. A GOS score of $\geq 4$ was categorized as a favorable outcome, and a GOS score of $\leq 3$ was considered an unfavorable outcome. 


\section{Statistical analysis}

The dependent outcome variable was dichotomized as dead versus alive and as unfavorable outcome (GOS 1-3) versus favorable outcome (GOS 4-5) [1]. GCS scores on admission (mean \pm standard deviation) were compared using the Mann-Whitney $U$ test in the different treatment groups. The chi-square test was used to compare the survival rate of the different groups. Binary logistic regression analysis was used to further determine the association between outcomes and prognostic indicators, including age, GCS on admission (mild, moderate, severe), pupillary reflexes, MLS, dominant-lesion volume, total lesion volume, cistern compression, traumatic subarachnoid hemorrhage, CT-visible deep lesions, type of predominant lesions, ICP monitoring, and type of treatment (conservative treatment, unilateral surgery, bilateral surgery) $[3,14,15]$. In the regression analysis, age, MLS, dominant-lesion volume, and total lesion volume were treated as continuous or nominal variables, and the remaining independent variables were treated as nominal variables. All data were analyzed with SPSS 16.0 (SPSS Inc., Chicago, IL, USA). Significance was defined by a two-tailed $\mathrm{P}$ value of less than 0.05 .

\section{Results}

A total of 80 consecutive patients with TABML were included in the retrospective study, the demographic, clinical, and outcome characteristics of whom patients are detailed in Table 1. Upon admission, 19 patients (23.8\%) had mild brain injuries (GCS 13-15), 14 (17.6\%) had moderate injuries (GCS 9-12), and 47 (58.8\%) had severe injuries (GCS 3-8). At 6 months after injury only three patients (3.8\%) had been lost to follow-up, leaving 77 patients with a GOS score $(2.9 \pm 1.6)$ available. The overall mortality was $31.3 \%$ and the rate of unfavorable outcomes was $56.3 \%$.

The major CT characteristics of TABML are shown in Table 2. Although the dominant-lesion volume $(37.6 \pm 21.6 \mathrm{ml})$ and total lesion volume $(51.9 \pm 28.4 \mathrm{ml})$ were large, the MLS was often mild $(4.0 \pm 3.4 \mathrm{~mm})$. SDH with or without $\mathrm{HC}$ was the most common type of predominant lesion, accounting for $42.5 \%$ of the total of 80 cases, with compression of basal cisterns, subarachnoid hemorrhage, and CT-visible deep lesions being frequently encountered.

Eighteen patients (22.5\%) underwent conservative treatment, 39 (48.8\%) unilateral surgery, and 23 (28.8\%) bilateral surgery. Bilateral surgery was performed simultaneously in 18 patients $(78.3 \%)$ and sequentially in five patients (21.7\%). The demographic, clinical, and outcome characteristics of the three treatment groups are shown in Tables 1 and 2. In the bilateral surgery group, patients were more likely to present with bilateral absence of pupillary response, large-volume lesions, complete cistern

Table 1 Baseline characteristics of the patients

\begin{tabular}{|c|c|c|c|c|}
\hline Characteristics & No. & Conservative & Unilateral & Bilateral \\
\hline No. of patients & 80 & 18 & 39 & 23 \\
\hline Male gender & $66(82.5)$ & $18(100.0)$ & $33(84.6)$ & $15(65.2)$ \\
\hline $\mathrm{Age}^{1}$ & $46.4(15.7)$ & $43.6(20.0)$ & $49.2(14.4)$ & $43.9(16.7)$ \\
\hline \multicolumn{5}{|l|}{ Cause of injury } \\
\hline Traffic & $42(52.5)$ & $10(55.6)$ & $15(38.5)$ & $17(73.9)$ \\
\hline Fall & $28(35.0)$ & $6(33.3)$ & $17(43.6)$ & $5(21.7)$ \\
\hline Violence & $4(5.0)$ & 0 & $4(10.3)$ & 0 \\
\hline Other/missing & $6(7.5)$ & $2(11.1)$ & $3(7.7)$ & $1(4.3)$ \\
\hline GCS score on admission ${ }^{1}$ & $8.5(3.8)$ & $9.2(4.3)$ & $9.2(3.7)$ & $6.7(3.3)$ \\
\hline AIS-head ${ }^{1}$ & $4.4(0.8)$ & $4.2(0.9)$ & $4.3(0.8)$ & $4.7(0.6)$ \\
\hline \multicolumn{5}{|l|}{ Pupillary response } \\
\hline Present & $52(66.3)$ & $11(61.1)$ & $31(79.5)$ & $10(43.5)$ \\
\hline One absent & $6(7.5)$ & $1(5.6)$ & $2(5.1)$ & $3(13.0)$ \\
\hline Bilateral absent & $21(26.3)$ & $6(33.3)$ & $5(12.8)$ & $10(43.5)$ \\
\hline \multicolumn{5}{|l|}{ Outcome } \\
\hline $\mathrm{GOS}^{1}$ & $2.9(1.6)$ & $2.6(2.0)$ & $3.3(1.5)$ & $2.5(1.4)$ \\
\hline Death & $25(31.3)$ & $10(55.6)$ & $7(17.9)$ & $8(34.8)$ \\
\hline Unfavorable (GOS score 1-3) & $45(56.3)$ & $12(66.7)$ & $16(41.0)$ & $17(73.9)$ \\
\hline Missing & $3(3.8)$ & $1(5.6)$ & $2(5.1)$ & 0 \\
\hline
\end{tabular}

${ }^{1}$ Mean (SD); All other variables: number (\%).

GCS, Glasgow Coma Scale; AIS, Abbreviated Injury Scale; GOS, Glasgow Outcome Scale; SD, standard deviation. 
Table 2 CT characteristics on admission

\begin{tabular}{|c|c|c|c|c|}
\hline CT characteristics & No. $(n=80)$ & Conservative $(n=18)$ & Unilateral $(n=39)$ & Bilateral $(n=23)$ \\
\hline \multicolumn{5}{|l|}{ Midline shift } \\
\hline Presence & $67(83.8)$ & $14(77.8)$ & $33(84.6)$ & $20(87.0)$ \\
\hline Mean, $\mathrm{mm}^{1}$ & $4.0(3.4)$ & $3.6(3.9)$ & $4.0(3.0)$ & $4.1(3.9)$ \\
\hline Volume dominant lesion, $\mathrm{mL}^{1}$ & $37.6(21.6)$ & $27.6(15.4)$ & $39.4(18.8)$ & $42.3(27.6)$ \\
\hline Total volume lesions, $\mathrm{mL}^{1}$ & $51.9(28.4)$ & $41.2(23.2)$ & $52.4(27.6)$ & $59.1(35.5)$ \\
\hline \multicolumn{5}{|l|}{ Dominant lesion } \\
\hline $\mathrm{EDH}$ & $17(21.3)$ & $1(5.6)$ & $10(25.6)$ & $6(26.1)$ \\
\hline $\mathrm{SDH}$ & $34(42.5)$ & $7(38.9)$ & $18(46.2)$ & $9(39.1)$ \\
\hline $\mathrm{HC}$ & $29(36.3)$ & $10(55.6)$ & $11(28.2)$ & $8(34.8)$ \\
\hline \multicolumn{5}{|l|}{ Compression of basal cisterns } \\
\hline Open & $19(23.8)$ & $6(33.3)$ & $12(30.8)$ & $1(4.3)$ \\
\hline Partially closed & $20(25.0)$ & $2(11.1)$ & $13(33.3)$ & $5(21.8)$ \\
\hline Completely closed & $41(51.3)$ & $10(55.6)$ & $14(35.9)$ & $17(73.9)$ \\
\hline Subarachnoid hemorrhage & $69(86.3)$ & $14(77.8)$ & $34(87.2)$ & $21(91.3)$ \\
\hline CT visible deep lesions & $26(32.5)$ & $7(38.9)$ & $8(20.5)$ & $11(47.8)$ \\
\hline
\end{tabular}

${ }^{1}$ Mean (SD); All other variables: number (\%).

$\mathrm{CT}$, computed tomography; $\mathrm{EDH}$, epidural hematoma; SDH, subdural hematoma; $(\mathrm{HC})$ Hemorrhagic contusion.

compression, and CT-visible deep lesions. Of the 15 patients with a GCS of 3-5 who underwent surgery, eight $(53.3 \%)$ died, four $(26.7 \%)$ survived in a vegetative state, and the remaining three $(20.0 \%)$ survived with severe disability. However, of the 47 surgical patients with a GCS score $\geq 6$, only seven (14.9\%) died, three (6.4\%) survived in a vegetative state, and eight (17.0\%) survived with severe disability.

We found that the absence of pupillary response, the compression of basal cisterns, and the type of treatment were statistically significant predictors of death; the worst GCS score on admission and the compression of basal cisterns both correlated significantly with an unfavorable outcome (Tables 3 and 4). Although the mortality and percentage of patients with an unfavorable outcome were higher in patients with a more severe MLS (Figure 1), as a continuous and nominal variable, MLS did not correlate with the outcomes significantly. Other factors (e.g., the type of dominant lesion) also did not correlate with the observed outcomes.

Table 3 Result of the binary logistic regression analysis for several characteristics and death

\begin{tabular}{llll}
\hline \multirow{2}{*}{ Characteristics } & \multicolumn{3}{l}{ Death $(\mathbf{n}=\mathbf{2 5})$} \\
\cline { 2 - 4 } Pupillary response & OR & $\mathbf{9 5 \%} \mathbf{C l}$ & $\mathbf{P}$ \\
\hline Compression of basal cisterns & 3.16 & $1.38,7.25$ & 0.007 \\
Type of treatment & 0.32 & $1.04,10.12$ & 0.043 \\
\hline OR & $0.13,0.84$ & 0.020 \\
\hline
\end{tabular}

OR, odds ratio; $\mathrm{Cl}$, confidence interval.

\section{Discussion}

Our study showed that the majority of patients (58.8\%) with TABML had severe brain injury. Conservative treatment was more likely associated with higher mortality and treatment failure. Unilateral or bilateral surgery, however, effectively lowered mortality in patients with TABML, especially in those with a GCS score $<6$. We also found that the GCS score, the pupillary reactivity, and the compression of basal cisterns are important prognostic predictors in TABML. Although MLS is an important prognostic predictor in TBI, our study revealed that it is not an outcome predictor in TABML $[3,15]$.

From this retrospective study, we cannot simply draw a conclusion that unilateral surgery is better than bilateral surgery based on the mortality alone, because the baseline characteristics of the two groups are not comparable. According to GCS score, the most important predictor of the severity of TBI, we found that bilateral surgery was commonly performed in patients with more severe injuries in comparison with those who underwent unilateral surgery (GCS, $6.7 \pm 3.3$ VS $9.2 \pm 3.7 ; \mathrm{p}<0.05$ ). Although patients who underwent conservative treatment

Table 4 Result of the binary logistic regression analysis for several characteristics and unfavorable outcome

\begin{tabular}{llll}
\hline \multirow{2}{*}{ Characteristics } & \multicolumn{4}{l}{ Unfavorable outcome $(\mathbf{n}=\mathbf{4 5})$} \\
\cline { 2 - 4 } GCS & OR & $\mathbf{9 5 \%} \mathbf{C l}$ & $\mathbf{P}$ \\
\hline Compression of basal cisterns & 2.74 & $1.17,6.42$ & 0.000 \\
\hline
\end{tabular}

OR, odds ratio; $\mathrm{Cl}$, confidence interval; GCS, Glasgow Coma Scale. 


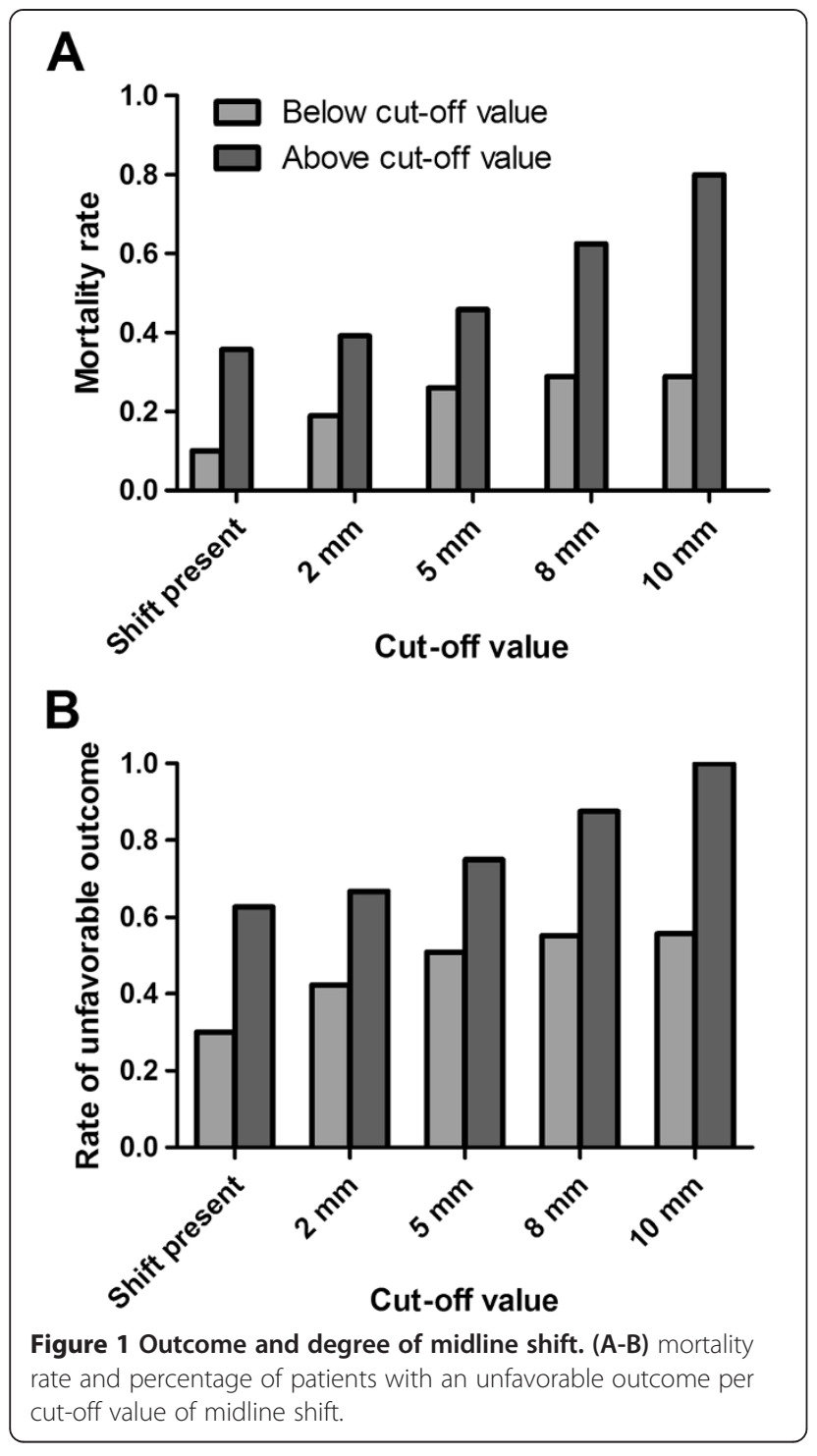

had higher GCS scores (GCS, $9.2 \pm 4.3$ VS $6.7 \pm 3.3$; $\mathrm{p}<0.05)$, a lower survival rate $(44.4 \%$ VS $65.2 \%$; $<<0.05)$ was found in comparison with bilateral surgery, indicating that bilateral surgery may be a life-saving procedure for TABML patients [5,10]. Logistic regression analysis, which adjusted the confounding factors, also confirmed that conservative treatment was related to higher mortality (odds ratio, 0.32; 95\% confidence interval, 0.13-0.84).

Our results are consistent with those of several previous studies demonstrating that bilateral surgery is an effective procedure in reducing mortality in patients with TABML $[5,10,17]$. Razack et al. reported a relatively low mortality $(20 \%)$ in patients with GCS scores of $8.8 \pm 0.82$ undergoing early simultaneous bilateral craniotomy, attributing the excellent results to the aggressive surgical intervention [5]. Walcott et al. studied a fatal subtype of TABML, bilateral acute SDH, and found that bilateral hemicraniectomy resulted in 33.3\% (3 of 9) of patients surviving in-hospital course [10]. This result may be due to surgical decompression being able to reduce intracranial hypertension more efficiently, with shorter duration of intracranial hypertension and fewer interventions $[13,17,18]$. Bilateral decompression has also been reported in patients with bilateral diffuse brain swelling, resulting in an equal mortality and a higher rate of unfavorable outcome in comparison with conservative treatment in a recent randomized trial [18]. The main explanation for the different results between this trial and our study is that TABML represents a more severe entity that is associated with poorer neurological status, higher ICP, and worse outcome $[4,8,18,19]$. Therefore, conservative treatment is always associated with poor ICP control and treatment failure, as revealed in our series, and aggressive surgical intervention is advocated.

Although bilateral surgery effectively lowered the mortality in TABML, a subset of patients did not benefit from such aggressive intervention $[5,10,19]$. Razack et al. reported five patients with a GCS of 3-5 undergoing bilateral surgery, resulting in $40 \%$ mortality and $0 \%$ favorable outcome [5]. Walcott et al. found that the in-hospital mortality was $100 \%$ in six patients with a GCS of 3-5, and the remaining three patients with a GCS of 6-8 survived [10]. Ucar et al. found that patients with severe TBI with initial GCS scores of 6-8 benefit from decompressive craniectomy, whereas those with GCS scores of $\leq 5$ do not [20]. We agree that patients with an advanced age, a GCS of 3, and bilateral fixed and dilated pupils are strongly identified with a fatal outcome [19]. Our study further confirmed that a GCS score of 3-5 was associated with a bad outcome $(53.3 \%$ mortality and $0 \%$ favorable outcome) even though the aggressive surgical intervention was applied, and we suggest that the aggressive surgery is warranted in patients with a GCS score of $\geq 6[5,10,13,19,20]$.

Our study also demonstrated that the outcome of patients with TABML correlated well with their initial GCS scores and pupillary reactivity. Other studies of TABML have also shown that GCS score was associated with outcome $[5,12]$. A study of multiple posttraumatic intracranial lesions found that both a low GCS score and a poor pupillary response predicted a bad outcome [7]. However, because of the early sedation and intubation, the prognostic value of early accessible clinical features (e.g., GCS and pupillary reactivity) is restricted $[10,14]$. The status of basal cisterns, an independent predictor unaffected by sedation, is directly related to outcome, as revealed in our study $[15,17]$. The fact that the obliteration of basal cisterns was associated with high mortality had previously been demonstrated in patients with bilateral lesions $[5,7,12]$. Taken together, these preoperative prognostic factors, particularly the status of basal cisterns, should be considered as the indications for early aggressive therapy in TABML $[5,21]$. 
More recent studies have revealed that MLS is important in predicting outcome and improving therapeutic decisionmaking $[3,15]$. We studied whether MLS can be useful in predicting outcomes in patients with TABML, and found that it did not correlate with the outcome $[5,7,9,10]$. The MLS represents pressure difference, which could be absent or mild in TABML because of the counter-mass effect $[4,5,7,12]$. Then patients may experience intracranial hypertension without obvious MLS being visible on CT. Therefore, when treating patients with TABML, the prognostic value of the MLS might be limited [3,15,22].

\section{Conclusions}

TABML is suggestive of severe brain injury with high mortality. Conservative therapy is always associated with treatment failure. However, surgical intervention can lower the mortality effectively and is advocated, especially in patients with a GCS score of at least 6. Although the baseline characteristics of the two surgical groups discussed herein are not comparable, bilateral surgery was always performed in patients with more severe injuries, and is a life-saving option. In treating TABML we should emphasize the importance of the GCS score, the pupillary reactivity, and, particularly, the compression of basal cisterns in outcomes prediction.

\section{Abbreviations \\ TABML: Traumatic acute bilateral mass-occupying lesions; GOS: Glasgow Outcome Scale; TBI: Traumatic brain injury; EDH: Epidural hematoma; SDH: Subdural hematoma; HC: Hemorrhagic contusion; CT: Computed tomography; DNR: Do Not Resuscitate; GOS: Glasgow Outcome Scale; ICP: Intracranial pressure.}

\section{Competing interests}

The authors declare that they have no competing interests.

\section{Authors' contributions}

$\mathrm{YH}, \mathrm{HS}$ and $\mathrm{CHY}$ conceived and designed the study. YH, HS, YQY, QL and KLL were responsible for data collection and writing the article. SQH, JS and $\mathrm{CHY}$ have made major contributions to analysis and interpretation. All authors have read, edited, and approved the final manuscript.

\section{Author details}

${ }^{1}$ Department of Neurosurgery, West China Hospital, Sichuan University, No 37, Guo Xue Xiang, Chengdu, Sichuan Province, China. ${ }^{2}$ Department of Orthopedics, Hospital of Chengdu Office People's Government of Tibetan Autonomous Region, Chengdu, Sichuan Province, China. ${ }^{3}$ Department of Neurosurgery, Xindu District People's Hospital of Chengdu, Chengdu, Sichuan Province, China.

Received: 20 August 2014 Accepted: 15 January 2015

Published: 24 January 2015

\section{References}

1. Maas Al, Stocchetti N, Bullock R. Moderate and severe traumatic brain injury in adults. Lancet Neurol. 2008;7(8):728-41.

2. Saatman KE, Duhaime AC, Bullock R, Maas Al, Valadka A, Manley GT. Classification of traumatic brain injury for targeted therapies. J Neurotrauma. 2008;25(7):719-38.

3. Jacobs B, Beems T, van der Vliet TM, Diaz-Arrastia RR, Borm GF, Vos PE. Computed tomography and outcome in moderate and severe traumatic brain injury: hematoma volume and midline shift revisited. J Neurotrauma. 2011;28(2):203-15.
4. Lobato RD, Cordobes F, Rivas JJ, de la Fuente M, Montero A, Barcena A, et al. Outcome from severe head injury related to the type of intracranial lesion. A computerized tomography study. J Neurosurg. 1983;59(5):762-74.

5. Razack N, Singh RV, Petrin D, Villanueva P, Green BA. Bilateral craniotomies for blunt head trauma. J Trauma. 1997;43(5):840-3.

6. Eisenberg HM, Gary Jr HE, Aldrich EF, Saydjari C, Turner B, Foulkes MA, et al. Initial CT findings in 753 patients with severe head injury. A report from the NIH Traumatic Coma Data Bank. J Neurosurg. 1990;73(5):688-98.

7. Caroli M, Locatelli M, Campanella R, Balbi S, Martinelli F, Arienta C. Multiple intracranial lesions in head injury: clinical considerations, prognostic factors, management, and results in 95 patients. Surg Neurol. 2001;56(2):82-8.

8. Sweet RC, Miller JD, Lipper M, Kishore PR, Becker DP. Significance of bilateral abnormalities on the CT scan in patients with severe head injury. Neurosurgery. 1978;3(1):16-21.

9. Frank E, Berger TS, Tew Jr JM. Bilateral epidural hematomas. Surg Neurol. 1982;17(3):218-22.

10. Walcott BP, Nahed BV, Sheth SA, Yanamadala V, Caracci JR, Asaad WF. Bilateral hemicraniectomy in non-penetrating traumatic brain injury. J Neurotrauma. 2012;29(10):1879-85.

11. Wong GK, Tang BY, Yeung JH, Collins G, Rainer T, Ng SC, et al. Traumatic intracerebral haemorrhage: is the CT pattern related to outcome? $\mathrm{Br} J$ Neurosurg. 2009;23(6):601-5.

12. Jayakumar PN, Sastry Kolluri VR, Basavakumar DG, Subbakrishna DK, Arya BY, Das BS, et al. Prognosis in contre-coup intracranial haematomas-a clinical and radiological study of 63 patients. Acta Neurochir (Wien). 1991;108(1-2):30-3.

13. Akyuz M, Ucar T, Acikbas C, Kazan S, Yilmaz M, Tuncer R. Effect of early bilateral decompressive craniectomy on outcome for severe traumatic brain injury. Turk Neurosurg. 2010;20(3):382-9.

14. Hukkelhoven CW, Steyerberg EW, Habbema JD, Farace E, Marmarou A, Murray GD, et al. Predicting outcome after traumatic brain injury: development and validation of a prognostic score based on admission characteristics. J Neurotrauma. 2005;22(10):1025-39.

15. Nelson DW, Nystrom H, MacCallum RM, Thornquist B, Lilja A, Bellander BM, et al. Extended analysis of early computed tomography scans of traumatic brain injured patients and relations to outcome. J Neurotrauma. 2010;27 (1):51-64.

16. Brain Trauma Foundation. Guidelines for the Management of Severe Traumatic Brain Injury. 3rd ed. New York: Brain Trauma Foundation; 2007. p. S1-59.

17. Bao YH, Liang YM, Gao GY, Pan YH, Luo QZ, Jiang JY. Bilateral decompressive craniectomy for patients with malignant diffuse brain swelling after severe traumatic brain injury: a 37-case study. J Neurotrauma. 2010;27(2):341-7.

18. Cooper DJ, Rosenfeld JV, Murray L, Arabi YM, Davies AR, D'Urso P, et al. Decompressive craniectomy in diffuse traumatic brain injury. N Engl J Med. 2011;364(16):1493-502

19. Ucar T, Akyuz M, Kazan S, Tuncer R. Role of decompressive surgery in the management of severe head injuries: prognostic factors and patient selection. J Neurotrauma. 2005;22(11):1311-8.

20. Yatsushige H, Takasato Y, Masaoka H, Hayakawa T, Otani N, Yoshino Y, et al. Prognosis for severe traumatic brain injury patients treated with bilateral decompressive craniectomy. Acta Neurochir Suppl. 2010;106:265-70.

21. Toutant SM, Klauber MR, Marshall LF, Toole BM, Bowers SA, Seelig JM, et al. Absent or compressed basal cisterns on first CT scan: ominous predictors of outcome in severe head injury. J Neurosurg. 1984;61(4):691-4.

22. Maas Al, Steyerberg EW, Butcher I, Dammers R, Lu J, Marmarou A, et al. Prognostic value of computerized tomography scan characteristics in traumatic brain injury: results from the IMPACT study. J Neurotrauma. 2007;24(2):303-14.

doi:10.1186/1471-2482-15-6

Cite this article as: Hu et al: Acute bilateral mass-occupying lesions in non-penetrating traumatic brain injury: a retrospective study. BMC Surgery $2015: 6$. 